\title{
Validity and Reliability of the Japanese Version of the Fertility Quality of Life (FertiQoL) Tool for Couples Undergoing Fertility Treatment
}

\author{
Kyoko Asazawa', Mina Jitsuzaki ${ }^{2}$, Akiko Mori ${ }^{3}$, Tomohiko Ichikawa4, Katsuko Shinozaki ${ }^{5}$, \\ Atsumi Yoshida6, Masami Kawanami6, Hiroshi Kamiyama7 \\ ${ }^{1}$ Department of Nursing, Tokyo Healthcare University, Tokyo, Japan \\ ${ }^{2}$ Department of Nursing, University of Occupational and Environmental Health, Fukuoka, Japan \\ ${ }^{3}$ Women's Health and Midwifery, Graduate School, St. Luke's International University, Tokyo, Japan \\ ${ }^{4}$ Graduate School of Medicine, Chiba University, Chiba, Japan \\ ${ }^{5}$ Graduate School of Health and Welfare Science, International University of Health and Welfare, Fukuoka, Japan \\ ${ }^{6}$ The Reproduction Center, Kiba Park Clinic, Tokyo, Japan \\ ${ }^{7}$ The Reproduction Center, Shibakoen Kamiyama Clinic, Tokyo, Japan \\ Email: 11DN001x2U-ts@slcn.ac.jp
}

How to cite this paper: Asazawa, K., Jitsuzaki, M., Mori, A., Ichikawa, T., Shinozaki, K., Yoshida, A., Kawanami, M. and Kamiyama, H. (2018) Validity and Reliability of the Japanese Version of the Fertility Quality of Life (FertiQoL) Tool for Couples Undergoing Fertility Treatment. Open Journal of Nursing, 8, 616-628. https://doi.org/10.4236/ojn.2018.89046

Received: August 15, 2018

Accepted: September 8, 2018

Published: September 11, 2018

Copyright $\odot 2018$ by authors and Scientific Research Publishing Inc. This work is licensed under the Creative Commons Attribution International License (CC BY 4.0).

http://creativecommons.org/licenses/by/4.0/

(c) (i) Open Access

\begin{abstract}
Background: FertiQoL, which measures the Quality of Life (QOL) of reproductive partners, has been translated and used in 45 languages in the world. The reliability and validity of the original English version of FertiQoL have been confirmed. However, there is still no report on the reliability and validity of the Japanese version by a large-scale survey. This study aimed to examine the reliability and validity of the Japanese version of the FertiQoL scale for measuring the QOL of patients with reproductive problems. Methods: An unsigned self-filled questionnaire survey was conducted in patients undergoing infertility treatment at seven facilities in the Kanto area in Japan using the 34 items of the Japanese version of the FertiQoL scale. The study design was quantitative cross-sectional descriptive research. The investigation period was from April 2013 to April 2018. The contents of the investigation were attributes, FertiQoL scale, and distress scale. To determine the construct validity, principal component analysis, confirmatory factor analysis, and correlation analysis for each subscale were performed using SPSS Statistics Ver. 23.0 and AMOS Ver. 23.0. The study was performed after obtaining approval from the Research Ethics Review Committee of the responsible institutions to which the researchers belong as an ethical consideration. Results: The participants included 1201 patients undergoing infertility treatment and who provided valid responses. The Cronbach's $\alpha$ was 0.92 , and confirmatory factor analysis identified six domains with 34 items that showed the following values: goodness of fit index $=0.877$, adjusted goodness of fit index $=0.855$,
\end{abstract}


comparative fit index $=0.893$, and root mean square error of approximation $=0.059$. The correlation coefficient was $0.669(\mathrm{p}<0.001)$ with the distress scale. Conclusions: The Japanese version of FertiQoL showed adequate reliability and validity for assessing the QOL of patients with reproductive problems in Japan.

\section{Keywords}

Infertility, QOL, Confirmatory Factor Analysis, Reliability, Validity

\section{Introduction}

In 2015, there were approximately 424,000 recorded patients receiving treatment using assisted reproductive technology (ART) in Japan, with about $5.1 \%$ of the total number of births born by ART [1]. Moreover, both the number of births from infertility treatment and the number of estimated patients have also increased [2]. Nevertheless, patients who undergo infertility treatment suffer from stress because of the physical burden of the treatment, reducing their quality of life (QOL) [3]. Qualitative studies on the psychological consequences of infertility describe infertility as a devastating experience, particularly for women [4] [5]. Women who are undergoing infertility treatment have significantly greater mental suffering than men in terms of stress, anxiety, and depression [6]. In particular, women suffer from mental and economic burdens, as well as marital deterioration [7].

QOL in reproduction has been shown to be negatively correlated with distress, which needs to be reduced to improve QOL [8]. Maintaining the QOL of people undergoing infertility treatment is of paramount importance, and providing quality care that takes QOL into account is necessary as a comprehensive approach in clinical practice [9]. Therefore, an intervention that takes into consideration reduction of stress on both men and women and improvement of their QOL is necessary.

As for the current status of intervention studies, stress management has been implemented in infertile women in Japan, and this has been shown to improve their QOL [10]. In addition, intervention studies for improving the QOL of infertile couples are being conducted in other countries, and counseling and educational programs have been shown to be effective [11].

Measurement of the QOL of infertile patients is based on the SF 36 [12] [13], WHOQOL [14], EuroQOL (EQ - 5 D) [15], and Health Utilities Index (HUI) [16], which have been used in previous studies as existing scales. However, these are comprehensive quality of life measurement tools in health and cannot be used to strictly measure the QOL of people with reproductive problems.

Therefore, Boivin et al. (2011) of Cardiff University in the UK developed the Fertility-related Quality of Life (FertiQoL) tool [17]. The validity and reliability of the English version of FertiQoL have been confirmed. The original version of 
FertiQoL is currently translated into 45 languages and can be used as a disease-specific QOL scale. The face and content validities of the translated Japanese version were previously examined by Shiro Hirayama, Tomoko Ogura, Akiko Mori, and Masako Ishikawa Coxal who made up the FertiQoL Team [18]. However, the results of their large-scale survey regarding the reliability and validity of the Japanese version of FertiQoL were not completely clear.

The aims of this study were 1) to verify the reliability and construct validity of the Japanese version of FertiQoL when used in quantitative surveys of men and women during infertility treatment, and 2) to examine its concurrent validity in terms of relationship with distress.

\section{Methods}

\subsection{Design}

Following the aims of this study, a quantitative cross-sectional descriptive questionnaire survey design was used.

\subsection{Definition of Terms}

QOL: Quality of life of men and women with reproductive problems

Distress: Therapy-induced mental suffering in the forms of stress, anxiety, and depression in men and women undergoing infertility treatments

\subsection{Participants and Setting}

The survey covered seven facilities consisting of a general hospital or a specialized infertility treatment facility that performs infertility treatment in Japan. These seven infertility treatment facilities were located in the Kanto area of Japan. They were chosen as their primary physicians and nursing directors agreed to cooperate with this study and the facilities helped in the data collection by purposive sampling of participants from a convenience sample.

The selection criteria included outpatients who were receiving infertility treatment and men and women who can read and write Japanese. When infertile patients had mental illness or serious disease in the past, they were excluded considering the additional burden of this study. The sample size was calculated as 1700 participants. The number of subjects necessary for factor analysis was 5 to 10 times the variables [19]. The number of people required for the survey using the 34 items of FertiQoL (Japanese version) was 340 based on an assumed recovery rate of $20 \%$.

\subsection{Procedures}

The nurse managers of the infertility treatment facilities cooperating with this study provided assistance in recruiting the participants by convenience sampling. The nurse managers and authors initially confirmed that all the participants met the inclusion criteria. The nurse managers then introduced the infertility patients to the authors. After obtaining permission from the infertility 
treatment facilities, verbal and written information regarding the research project was provided to the participants. A self-administered questionnaire was distributed to the participants either as a couple or as a single partner. Submission of the completed questionnaire was considered as indicating consent from the participant. Each participant was asked to return the completed questionnaire in a sealed envelope either by post or by placing it in a collection box at the waiting lounge of the treatment facility. During the study period from April 2013 to April 2018, 1867 questionnaires were distributed to eligible patients. A total of $1243(66.6 \%)$ completed questionnaires were returned, of which 1201 $(64.3 \%)$ were suitable for analyses.

\subsection{Survey Contents}

\subsubsection{Participants' Characteristics}

The participants' characteristics included age, length of marriage, duration of infertility, infertility treatment period, significant medical history, significant gynecological disease, marital status, with or without of children, cause of infertility, nature of the current treatment, and frequency of changing hospitals/clinics.

\subsubsection{Quality of Life}

The FertiQoL tool developed by Boivin et al. (2011) [17] was used for evaluating the QOL of men and women in relation to their personal experiences of fertility problems. The original version of FertiQoL has been translated into 45 languages (Cardiff Fertility Research Group, n.d.). The Japanese version of FertiQoL was used in the present study. FertiQoL consists of 34 items with five response categories ranging from 0 (lower QOL) to 4 (higher QOL). A higher score on the total FertiQoL scale or one of the subscales (range 0 - 100) indicates a better QOL (Boivin et al., 2011). The scaled scores for the subscale and total scales are computed by multiplying the relevant raw score by $25 / 34$. These items include six subscales: 1) emotional, 2) mind/body, 3) relational, 4) social, 5) environment, and 6) tolerability. FertiQoL assesses the effects of fertility problems on diverse life areas, namely, self-esteem, emotions, general health, partnership, family and social relationships, work life, and future life plans; the optional FertiQoL treatment factor scale assesses the burden/tolerability of the fertility treatment. Higher scores indicate a higher QOL. Boivin et al. (2011) reported the Cronbach's $\alpha$ coefficient of FertiQoL to be $0.72-0.92$ for 109 men and 1305 women with fertility problems from six countries.

\subsubsection{Distress}

The psychological distress of infertile couples was previously evaluated using the Japanese version of the distress scale developed by Asazawa \& Mori (2015) [20]. This distress scale is a three-item inventory consisting of the following questions: 1) "Do you feel stressed by the treatment?", 2) "Do you feel depressed because of the treatment?", and 3) "Do you feel any anxiety from the treatment?". The response categories ranged from 1 (no) to 5 (yes). Higher scores indicate the pres- 
ence of a higher distress. The instrument showed an acceptable reliability with a Cronbach's $\alpha$ coefficient of 0.89 , establishing its internal consistency, and experienced midwives have also established its face and content validities [20]. According to Aarts et al. (2011) [8], the Hospital Anxiety and Depression Scale (HADS) was used to examine the Dutch version of FertiQoL and its concurrent validity. In the present study, we used this distress scale whose number of items is small for measuring distress. The reliability and validity of this distress scale were reconfirmed.

\subsection{Statistical Analysis}

For data analyses, the following were performed using SPSS Ver. $23.0 \mathrm{~J}$ for Windows and AMOS Ver. 23 (SPSS, Chicago, IL, USA). In all analyses, p-values $<0.05$ were considered to indicate a statistically significant difference:

1) Consideration of validity: Principal component analysis was conducted to determine the number of items and the nature of the underlying factors in the Japanese version of FertiQoL. The component loading amount and contribution rate were calculated for each of the six subscales of FertiQoL. Principal component analysis was used to verify the appropriateness of the questions included in each subscale.

2) Consideration of internal consistency: For confirmation of internal consistency, Cronbach's $\alpha$ for the entire scale of the Japanese version of FertiQoL and subscale was calculated.

3) Consideration of concurrent validity: Pearson's correlation coefficient was calculated using the Japanese version of FertiQoL and distress scale, and the concurrent validity was examined.

4) Examination of model fitness: The construct validity in the Japanese version of FertiQoL was examined using confirmatory factor analysis (CFA) to assess the model fitness. Various fit indices were used to assess the fitness of the model to the data as follows: goodness of fit index (GFI), adjusted goodness of fit index (AGFI), comparative fit index (CFI), and root mean square error of approximation (RMSEA).

\subsection{Ethical Approval}

The Ethics Committee for Epidemiological Studies of St Luke's International University and the Institutional Ethics Committee of Tokyo Healthcare University reviewed and provided ethical approval for this research project. The questionnaires were anonymous and the private information of each participant was not identified. All the questionnaires were completed by the participants themselves and then returned directly and individually to the researchers by post.

\section{Results}

\subsection{Characteristics of the Study Participants}

We received 1243 responses (response rate: 66.6\%) from the patients in whom 
we distributed the questionnaires to 1867 infertile participants. There were 42 responses that were excluded owing to missing data. Thus, 1201 responses were analyzed (effective response rate: $64.3 \%$ ). To confirm the reliability and validity of the Japanese version of FertiQoL for men and women, mixed data of the men and women were used.

Table 1 shows the demographic characteristics of the participants. The mean age was 37.2 (SD 5.1) years; 727 participants (60.5\%) were men and 474 participants $(39.5 \%)$ were women. Infertility was due to a male factor in $13.2 \%$ of the cases, a female factor in $21.6 \%$, both male and female factors in $13.2 \%$, and unknown factors in $13.9 \%$. The treatments were artificial insemination in $40.9 \%$, ART in $20.8 \%$, and timing therapy in $16.5 \%$. Of the participants, $10 \%$ had significant medical history, and $31.6 \%$ of the women had gynecological diseases such as uterine fibroids and endometriosis.

\subsection{Reliability of the Japanese Version of FertiQoL}

Principal component analysis was performed to determine whether the factor loading matrix possessed the six subscale structure of the original version in the Japanese version of FertiQoL. The principal component loadings of all the 34 items in the six subscale structure were "Emotional", "Mind/Body", "Relational", "Social", "Environment", and "Tolerability" which ranged from 0.33 to 0.83 , with $30.8 \%$ of the total variance explained (Table 2 ). The principal component loadings of the subscales "Emotional" and "Social" did not exceed the recommended value of 0.40 .

The overall Cronbach's $\alpha$ of the Japanese version of FertiQoL with 34 items was 0.92 , and ranged from 0.67 to 0.86 in the six subscales. Although the Cronbach's $\alpha$ of the "Relational" subscale was 0.67 which was $<0.7$, it was considered acceptable as it was a required subscale for this scale. The Cronbach's $\alpha$ for the distress scale was 0.90, which indicated excellent internal consistency. These calculations confirmed the reliability of the distress and FertiQoL subscales. Therefore, both scales showed internal consistency.

\subsection{Validity of the Japanese Version of FertiQoL}

For concurrent validity, the relationships among the Japanese version of the FertiQoL score, the six subscale score of FertiQoL, and the distress scale score were examined by determining Pearson's correlation coefficient. As shown in Table 3, the FertiQoL and distress scale scores showed a negative significant correlation of $r=0.669$ at the $1 \%$ level. The FertiQoL six subscale score and the distress scale score also showed a negative significant correlation at the $1 \%$ level. The correlation coefficient between the "Relational" subscale and the distress scale was $\mathrm{r}=$ -0.226 ( $p<0.001$ ), and the correlation coefficient between the "Environment" and the distress scale was $r=-0.193(p<0.001)$. Although it was a significant correlation, it had a low coefficient and weak correlation. However, the correlation coefficients between the FertiQoL "Emotional", "Mind/Body", "Social”, and 
"Tolerability" subscales and the distress scale were $\mathrm{r}=-0.50$ or more $(\mathrm{p}<0.001)$, and a significantly moderate correlation was confirmed.

Regarding the construct validity, the fitness of the model of the Japanese version of FertiQoL was examined using confirmatory factor analysis. The following fit indices were found: $\mathrm{GFI}=0.877, \mathrm{AGFI}=0.855, \mathrm{CFI}=0.893$, and RMSEA $=0.059$ (Figure 1). Although the GFI and CFI were 0.9 or less, the AGFI was 0.85 or more, and it met the criterion of GFI > AGFI. The RMSEA was 0.059 and it met the criterion of $<0.08$. Significant path coefficients were obtained in all the items between the latent variables and the observed variables. The model exactly fits the study population. These results showed that the Japanese version of FertiQoL was as unidimensional as the original version.

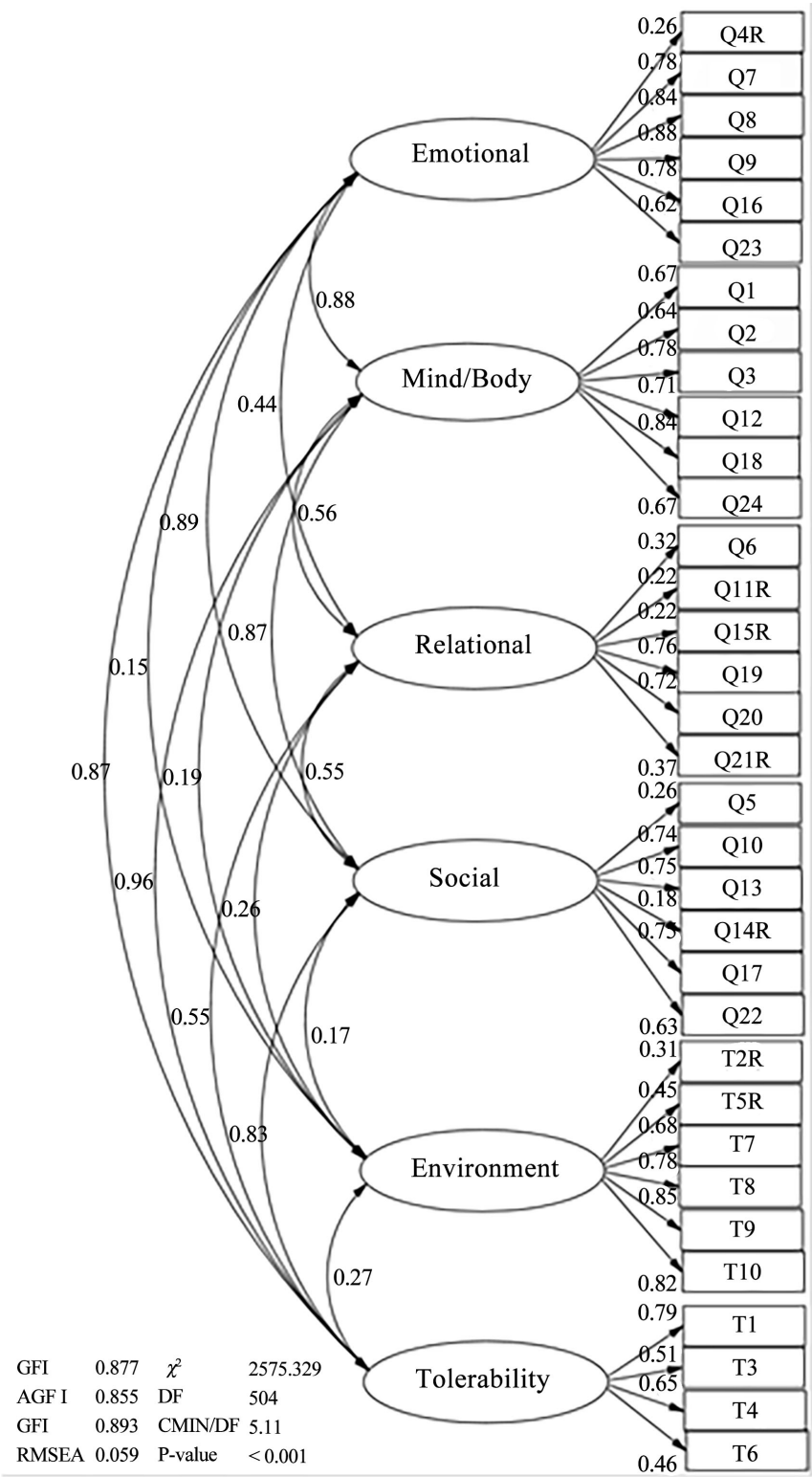

Figure 1. Results of the confirmatory factor analysis of the Japanese version of FertiQoL $(\mathrm{N}=1201)$. 
Table 1. Characteristics of the study participants $(\mathrm{N}=1201)$.

\begin{tabular}{|c|c|c|c|c|c|c|}
\hline \multirow{2}{*}{ Characteristics } & \multicolumn{2}{|c|}{ Participants $(\mathrm{N}=1201)$} & \multicolumn{2}{|c|}{ Men $(\mathrm{n}=727)$} & \multicolumn{2}{|c|}{ Women $(\mathrm{n}=474)$} \\
\hline & Mean & SD & Mean & $\mathrm{SD}$ & Mean & $\mathrm{SD}$ \\
\hline Age (years) & 37.2 & 5.1 & 37.9 & 5.3 & 36.2 & 4.5 \\
\hline Duration of marriage & 60.4 & 43.3 & 59.3 & 43.0 & 62.1 & 48.8 \\
\hline Duration of infertility & 42.7 & 37.9 & 41.2 & 37.5 & 45.0 & 38.5 \\
\hline \multirow[t]{2}{*}{ Duration of infertility treatment } & 17.6 & 19.4 & 17.1 & 19.4 & 18.3 & 19.5 \\
\hline & $\mathrm{n}$ & $\%$ & $\mathrm{n}$ & $\%$ & $\mathrm{n}$ & $\%$ \\
\hline \multicolumn{7}{|l|}{ Significant medical history } \\
\hline yes & 120 & 10.0 & 58 & 8.0 & 62 & 13.1 \\
\hline no & 1081 & 90.0 & 669 & 92.0 & 412 & 86.9 \\
\hline \multicolumn{7}{|l|}{ Significant gynecological disease } \\
\hline yes & 150 & 31.6 & 0 & 0.0 & 150 & 31.6 \\
\hline no & 324 & 68.4 & 0 & 0.0 & 324 & 68.4 \\
\hline \multicolumn{7}{|l|}{ Marriage status } \\
\hline first marriage & 1034 & 86.1 & 627 & 86.2 & 407 & 85.9 \\
\hline remarried & 157 & 13.1 & 94 & 12.9 & 63 & 13.3 \\
\hline not married & 8 & 0.7 & 5 & 0.7 & 3 & 0.6 \\
\hline no response & 2 & 0.2 & 1 & 0.1 & 1 & 0.2 \\
\hline \multicolumn{7}{|l|}{ Having a child } \\
\hline yes & 142 & 11.8 & 91 & 12.5 & 51 & 10.8 \\
\hline no & 1057 & 88.0 & 636 & 87.5 & 472 & 99.6 \\
\hline No response & 2 & 0.2 & 0 & 0.0 & 2 & 0.4 \\
\hline \multicolumn{7}{|l|}{ Causes of infertility } \\
\hline unexplained & 453 & 37.7 & 292 & 40.2 & 161 & 34.0 \\
\hline male factor & 158 & 13.2 & 92 & 12.7 & 66 & 13.9 \\
\hline female factor & 259 & 21.6 & 126 & 17.3 & 133 & 28.1 \\
\hline male and female factors & 158 & 13.2 & 96 & 13.2 & 62 & 13.1 \\
\hline unknown & 167 & 13.9 & 118 & 16.2 & 49 & 10.3 \\
\hline no response & 6 & 0.5 & 3 & 0.4 & 3 & 0.6 \\
\hline \multicolumn{7}{|l|}{ Type of treatment } \\
\hline under examination or undecided & 146 & 12.2 & 97 & 13.3 & 49 & 10.3 \\
\hline timing therapy & 198 & 16.5 & 123 & 16.9 & 75 & 15.8 \\
\hline ovulation-inducing drugs & 95 & 7.9 & 50 & 6.9 & 45 & 9.5 \\
\hline artificial insemination & 491 & 40.9 & 292 & 40.2 & 199 & 42.0 \\
\hline ART & 250 & 20.8 & 146 & 20.1 & 104 & 21.9 \\
\hline unknown & 17 & 1.4 & 17 & 2.3 & 0 & 0.0 \\
\hline no response & 4 & 0.3 & 2 & 0.3 & 2 & 0.4 \\
\hline \multicolumn{7}{|l|}{ Changing clinics } \\
\hline yes & 493 & 41.0 & 270 & 37.1 & 223 & 47.0 \\
\hline no & 705 & 58.7 & 457 & 62.9 & 248 & 52.3 \\
\hline no response & 3 & 0.2 & 0 & 0.0 & 3 & 0.6 \\
\hline
\end{tabular}

ART $=$ Assisted reproductive technology. 
Table 2. Confirmation of the reliability and validity of FertiQoL $(\mathrm{N}=1201)$.

\begin{tabular}{|c|c|c|c|c|c|c|c|}
\hline $\begin{array}{c}\text { Scale } \\
\text { Subscale }\end{array}$ & $\mathrm{N}$ & Mean & $\mathrm{SD}$ & Number of items & $\begin{array}{c}\text { Proportion of } \\
\text { variance }\end{array}$ & $\begin{array}{c}\text { Principal component } \\
\text { loading }\end{array}$ & $\begin{array}{c}\text { Cronbach's } \\
\alpha\end{array}$ \\
\hline Emotional & 1201 & 62.0 & 20.3 & 6 & 58.4 & $0.33-0.88$ & 0.86 \\
\hline Mind/Body & 1201 & 70.5 & 20.4 & 6 & 60.2 & $0.70-0.85$ & 0.86 \\
\hline Relational & 1201 & 73.2 & 14.9 & 6 & 38.0 & $0.47-0.72$ & 0.67 \\
\hline Social & 1201 & 67.2 & 17.8 & 6 & 45.3 & $0.34-0.82$ & 0.73 \\
\hline Environment & 1201 & 53.2 & 15.5 & 6 & 53.2 & $0.41-0.86$ & 0.77 \\
\hline Tolerability & 1201 & 68.7 & 21.6 & 4 & 59.7 & $0.70-0.86$ & 0.77 \\
\hline
\end{tabular}

Principal component analysis. ${ }^{*}$ The scaled scores for the subscale and total scales is computed by multiplying the relevant raw score by $25 / 34$.

Table 3. Association between FertiQoL and distress scale $(\mathrm{N}=1201)$.

\begin{tabular}{|c|c|c|c|c|c|c|c|c|c|c|c|c|c|c|}
\hline & \multicolumn{14}{|c|}{ FertiQoL and Subscale } \\
\hline & \multicolumn{2}{|c|}{$\begin{array}{l}\text { FertiQoL } \\
\text { score }\end{array}$} & \multicolumn{2}{|c|}{$\begin{array}{c}\text { Emotional } \\
\text { score }\end{array}$} & \multicolumn{2}{|c|}{$\begin{array}{l}\text { Mind/Body } \\
\text { score }\end{array}$} & \multicolumn{2}{|c|}{$\begin{array}{l}\text { Relational } \\
\text { score }\end{array}$} & \multicolumn{2}{|c|}{$\begin{array}{l}\text { Social } \\
\text { score }\end{array}$} & \multicolumn{2}{|c|}{$\begin{array}{l}\text { Environment } \\
\text { score }\end{array}$} & \multicolumn{2}{|c|}{$\begin{array}{c}\text { Tolerability } \\
\text { score }\end{array}$} \\
\hline $\begin{array}{c}\text { Distress } \\
\text { scale score }\end{array}$ & -0.669 & $* * *$ & -0.644 & $* * *$ & -0.661 & $* * *$ & -0.226 & $* * *$ & -0.502 & $* * *$ & -0.193 & $* * *$ & -0.636 & $* * *$ \\
\hline
\end{tabular}

Pearson's product moment correlation coefficient. ${ }^{* * *} \mathrm{p}<0.001$.

\section{Discussion}

\subsection{Validity and Reliability of the Japanese Version of FertiQoL}

This study aimed to examine the validity and reliability of the Japanese version of FertiQoL. The results indicate that the Japanese version of FertiQoL is a reliable and valid instrument for evaluating the QOL level of infertility patients and people with reproductive problems in Japan. The statistical evaluation of the Japanese version of FertiQoL was adequate because the distribution was appropriate for the parametric method. The reliability of the full Japanese version of FertiQoL was evaluated according to internal consistency, which was sufficient as indicated by a Cronbach's $\alpha$ of 0.92 .

The internal consistency of the "Relational" subscale was acceptable but was relatively low ( $\alpha=0.67)$. In previous studies, the Cronbach's $\alpha$ of the "Relational" subscale of FertiQoL was 0.80 for the original English version [17], 0.72 for the Dutch version [8], 0.20 for the Chinese version [21], and 0.64 for the Persian version [22]. In a previous study, there was a difference in the Cronbach's $\alpha$ depending on the country, race, and language of the study subject. It is desirable for the reliability coefficient to be 0.70 or more [23]. The Cronbach's $\alpha$ of the "Relational" subscale in the Japanese version of FertiQoL was 0.67. Although these values were somewhat lower, we considered these levels as acceptable.

The results of both principal component analysis and CFA indicated the appropriateness of the six-factor structure. The principal component loading of all the 34 items in the six subscale structure ranged from 0.33 to 0.83 . This range 
did not exceed the recommended value of 0.40 in the two subscales. Nevertheless, this range was considered acceptable from our judgment.

Concurrent validity was validated because a significant negative correlation was found between the FertiQoL score and the distress score. In the FertiQoL score and six subscale score, a weak negative correlation - strong negative correlation was observed for the distress scale score. From this result, a certain degree of concurrent validity was confirmed. Although the reliability and validity of the distress scale used for considering the concurrent validity have been confirmed, the use of scales such as HADS, which is frequently used globally, was better [24] [25]. For example, the QoL and anxiety and depression scores of the infertility patients were significantly negatively related when using FertiQoL and HADS [26] [27].

In addition, because the RMSEA was 0.58 , this was considered to be the best fit model. An RMSEA of $<0.08$ is recommended for an "acceptable model" [28]. The model exactly fits the study population. These results showed that the Japanese version of FertiQoL was as unidimensional as the original scale. Therefore, the Japanese version of FertiQoL is a measure with a certain reliability and validity. Also, as the model fit satisfies a certain criterion in the confirmatory factor analysis, it is determined that the model can be used clinically.

\subsection{Implications}

The QOL of Japanese patients during infertility treatment is particularly low compared with that of non-Japanese patients across the world. Among patients undergoing infertility treatment, Japanese patients had lower QOL scores than Italian [29], Romanian [30], and Dutch [31] patients. However, the QOL of Japanese patients was higher than that of Taiwanese patients [21]. Thus, FertiQoL can be used to prevent QOL decline during infertility treatment. In particular, it is necessary to grasp which domains of Emotional, Mind/Body, Relational, Social, Environment, and Tolerability are unsatisfactory and need support in patients undergoing infertility treatment. This information can be used for counseling and individual correspondence.

\subsection{Limitations and Future Challenges}

In this study, a questionnaire was distributed to either a couple or a single partner visiting the surveyed clinics. The study participants were about $60 \%$ men and $40 \%$ women. As the cause of infertility is considered to be $50 \%$ each for the man and women, their recruitment should be equal. When using this scale in the future, factors related to the QOL of men and women undergoing infertility treatment need to be carefully identified, and support that does not reduce QOL should be considered.

\section{Conclusion}

The validity and reliability of the Japanese version of FertiQoL consisting of 34 
item and six subscales were confirmed on the basis of the results of the construct validity, concurrent validity, and internal consistency. In the confirmatory factor analysis, the model fit of the scale was verified to be acceptable. Thus, FertiQoL is expected to be used for QOL evaluation to improve support and prevent deterioration of the QOL of patients with reproductive health problems.

\section{Acknowledgements}

The authors thank the patients who participated in this research, as well as the facility staff and research assistants.

The authors also thank Dr. Edward Barroga

(http://orcid.org/0000-0002-8920-2607) for reviewing and editing the manuscript.

The results of this study were presented at the 23rd Annual Conference of St. Luke's Society for Nursing Research in 2018.

\section{Conflicts of Interest}

The authors declare that there they have no conflicts of interest associated with this study.

\section{Disclosures}

Human rights statements and informed consent

All procedures were performed in accordance with the ethical standards of the responsible committees for human experimentation (institutional and national) and the 1964 Helsinki Declaration and later amendments. Informed consent was obtained from all patients.

\section{Animal studies}

Animal studies were not performed in this study.

\section{Approval by ethics committee}

The protocol of this research project was approved by the Ethics Committee of St. Luke's International University and Tokyo Healthcare University.

\section{References}

[1] Japan Society of Obstetrics \& Gynecology (2017) 2017 Ethics Committee Report, Heisei 28 year. http://fa.kyorin.co.jp/jsog/readPDF.php?file=69/9/069091841.pdf

[2] Crawford, S., Fussman, C., Bailey, M., Bernson, D., Jamieson, D.J., Murray-Jordan, M. and Kissin, D.M. (2015) Estimates of Lifetime Infertility from Three States: The Behavioral Risk Factor Surveillance System. Journal of Women's Health, 24, 578-586. https://doi.org/10.1089/jwh.2014.5102

[3] Boivin, J. and Schmidt L. (2005) Infertility-Related Stress in Men and Women Predicts Treatment Outcome 1 Year Later. Fertility and Sterility, 83, 1745-1752. https://doi.org/10.1016/j.fertnstert.2004.12.039

[4] Greil, A.L. (1997) Infertility and Psychological Distress: A Critical Review of the Literature. Social Science \& Medicine, 45, 1679-1704.

https://doi.org/10.1016/S0277-9536(97)00102-0 
[5] Niino and Okai (2008) A Study of Public Support for Patients Being Treated for Infertility: The First Report. Japanese Journal of Maternal Health, 49, 138-144. https://ci.nii.ac.jp/naid/110006656148

[6] Peterson, B.D., Newton, C.R., Rosen, K.H. and Skaggs, G.E. (2006) Gender Differences in How Men and Women Who Are Referred for IVF Cope with Infertility Stress. Human Reproduction, 21, 2443-2449.

https://doi.org/10.1093/humrep/del145

[7] Nishiwaki, M. (2000) Analysis of Stress Factors on Infertile Women under Medical Treatment. Bulletin of Yamanashi Medical University, 17, 48-51.

[8] Aarts, J.W., van Empel, I.W., Boivin, J., Nelen, W.L., Kremer, J.A. and Verhaak, C.M. (2011) Relationship between Quality of Life and Distress in Infertility: A Validation Study of the Dutch FertiQoL. Human Reproduction, 26, 1112-1118. https://doi.org/10.1093/humrep/der051

[9] Chachamovich, J.R., Chachamovich, E., Ezer, H., Fleck, M.P., Knauth, D. and Passos, E.P. (2010) Investigating Quality of Life and Health-Related Quality of Life in Infertility: A Systematic Review. Journal of Psychosomatic Obstetrics \& Gynecology, 31, 101-110. https://doi.org/10.3109/0167482X.2010.481337

[10] Mori, A. (2009) Supporting Stress Management for Women Undergoing the Early Stages of Fertility Treatment: A Cluster-Randomized Controlled Trial. Japan Journal of Nursing Science, 6, 37-49. https://doi.org/10.1111/j.1742-7924.2009.00119.x

[11] Boivin, J. (2003) A Review of Psychosocial Interventions in Infertility. Social Science \& Medicine, 57, 2325-2341. https://doi.org/10.1016/S0277-9536(03)00138-2

[12] Fukuhara, S., Ware Jr., J.E., Kosinski, M., Wada, S. and Gandek, B. (1998) Psychometric and Clinical Tests of Validity of the Japanese SF-36 Health Survey. Journal of Clinical Epidemiology, 51, 1045-1053. https://doi.org/10.1016/S0895-4356(98)00096-1

[13] Fukuhara, S., Bito, S., Green, J., Hsiao, A. and Kurokawa K. (1998) Translation, Adaptation, and Validation of the SF-36 Health Survey for Use in Japan. Journal of Clinical Epidemiology, 51, 1037-1044. https://doi.org/10.1016/S0895-4356(98)00095-X

[14] World Health Organization (1996) WHOQOL-BREF Introduction, Administration, Scoring and Generic Version of the Assessment. Field Trial Version. Resource Document. World Health Organization. http://www.who.int/mental_health/media/en/76.pdf

[15] EuroQol Group (1990) EuroQol-A New Facility for the Measurement of Health-Related Quality of Life. Health Policy, 16, 199-208.

https://doi.org/10.1016/0168-8510(90)90421-9

[16] Feeny, D., Furlong, W., Boyle, M. and Torrance, G.W. (1995) Multi-Attribute Health Status Classification Systems. Health Utilities Index. Pharmacoeconomics, 7, 490-502. https://doi.org/10.2165/00019053-199507060-00004

[17] Boivin, J., Takefman, J. and Braverman, A. (2011) The Fertility Quality of Life (FertiQoL) Tool: Development and General Psychometric Properties. Human Reproduction, 26, 2084-2091. https://doi.org/10.1093/humrep/der171

[18] Cardiff University (2018) Fertility Quality of Life Tool FertiQol Team. Contributions to FertiQoL Development Process. http://sites.cardiff.ac.uk/fertiqol/team/fertiqol-team/

[19] Arrindel, W.A. and van der Ende, J. (1985) An Empirical Test of the Utility of the Observations-to-Variables-Ratio in Factor and Components Analysis. Applied Psychological Measurement, 9, 165-178. 
https://doi.org/10.1177/014662168500900205

[20] Asazawa, K. and Mori, A. (2015) Development of a Partnership Causal Model for Couples Undergoing Fertility Treatment. Japan Journal of Nursing Science, 12, 208-221. https://doi.org/10.1111/jjns.12061

[21] Hsu, P.Y., Lin, M.W., Hwang, J.L., Lee, M.S. and Wu, M.H. (2013) The Fertility Quality of Life (FertiQoL) Questionnaire in Taiwanese Infertile Couples. Taiwanese Journal of Obstetrics \& Gynecology, 52, 204-209. https://doi.org/10.1016/j.tjog.2013.04.009

[22] Maroufizadeh, S., Ghaheri, A., Amini, P. and Samani, R.O. (2017) Psychometric Properties of the Fertility Quality of Life Instrument in Infertile Iranian Women. International Journal of Fertility \& Sterility, 10, 371-379. http://ijfs.ir/journal/article/abstract/4696

[23] Taber, K.S. (2017) The Use of Cronbach's Alpha When Developing and Reporting Research Instruments in Science Education. Research in Science Education, 1-24. https://link.springer.com/article/10.1007/s11165-016-9602-2 https://doi.org/10.1007/s11165-016-9602-2

[24] Zigmond, A.S. and Snaith, R.P. (1983) The Hospital Anxiety and Depression Scale. Acta Psychiatrica Scandinavica, 67, 361-370. https://doi.org/10.1111/j.1600-0447.1983.tb09716.x

[25] Kugaya, A., Akechi, T., Okuyama, T., Okamura, H. and Uchitomi, Y. (1998) Screening for Psychological Distress in Japanese Cancer Patients. Japanese Journal of Clinical Oncology, 28, 333-338. https://doi.org/10.1093/jjco/28.5.333

[26] Aarts, J.W., Huppelschoten, A.G., van Empel, I.W., Boivin, J., Verhaak, C.M., Kremer, J.A. and Nelen, W.L. (2012) How Patient-Centred Care Relates to Patients' Quality of Life and Distress: A Study in 427 Women Experiencing Infertility. $\mathrm{Hu}$ man Reproduction, 27, 488-495. https://doi.org/10.1093/humrep/der386

[27] Dural, O., Yasa, C., Keyif, B., Celiksoy, H., Demiral, I., Yuksel, O.B., Gungor, U.F. and Bastu, E. (2016) Effect of Infertility on Quality of Life of Women: A Validation Study of the Turkish FertiQoL. Human Fertility, 19, 186-191. https://doi.org/10.1080/14647273.2016.1214754

[28] MacCallum, R.C., Browne, M.W. and Sugawara, H.M. (1996) Power Analysis and Determination of Sample Size for Covariance Structure Modeling. Psychological Methods, 1, 130-149. http://psycnet.apa.org/buy/1996-04469-002 https://doi.org/10.1037/1082-989X.1.2.130

[29] Donarelli, Z., Lo, C.G., Gullo, S., Salerno, L., Marino, A., Sammartano, F. and Allegra, A. (2016) The Fertility Quality of Life (FertiQoL) Questionnaire Relational Subscale: Psychometric Properties and Discriminant Validity across Gender. $\mathrm{Hu}$ man Reproduction, 31, 2061-2071. https://doi.org/10.1093/humrep/dew168

[30] Cristiana, C.H. (2013) Gender Differences in Quality of Life, Intensity of Dysfunctional Attitudes, Unconditional Self-Acceptance, Emotional Distress and Dyadic Adjustment of Infertile Couples. Procedia-Social and Behavioral Sciences, 78, 506-510. https://doi.org/10.1016/j.sbspro.2013.04.340

[31] Huppelschoten, A.G., van Dongen, A.J., Verhaak, C.M., Smeenk, J.M., Kremer, J.A. and Nelen, W.L. (2013) Differences in Quality of Life and Emotional Status between Infertile Women and Their Partners. Human Reproduction, 28, 2168-2176. https://doi.org/10.1093/humrep/det239 